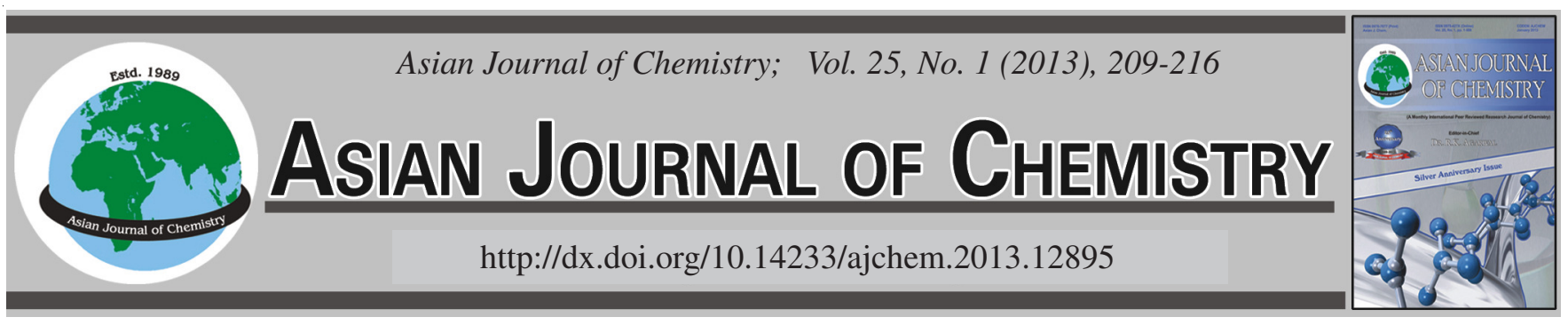

\title{
Spectral and Thermal Studies of Transition Metal Complexes of Acetamido Benzoic Acids with Hydrazine
}

\author{
E. Helen Pricilla Bai ${ }^{1}$ and S. Vairam ${ }^{2, *}$
}

${ }^{1}$ Department of Chemistry, Park College of Engineering and Technology, Coimbatore-641 659, India

${ }^{2}$ Department of Chemistry, Government College of Technology, Coimbatore-641 013, India

*Corresponding author: E-mail: vamshen@yahoo.com

\begin{abstract}
The metal complexes of isomeric acetamido benzoic acids with $\mathrm{Co}, \mathrm{Ni}, \mathrm{Zn}$ and $\mathrm{Cd}$ metals have been prepared and characterized by analytical, spectroscopic (IR, UV reflectance), micro elemental analysis, simultaneous TG-DTA, powder XRD, magnetic susceptibility measurements and SEM-EDS studies: [Ni(2-acamb $\left.)_{2}\left(\mathrm{~N}_{2} \mathrm{H}_{4}\right)\right] \cdot 2 \mathrm{H}_{2} \mathrm{O}$; $\left[\mathrm{M}(2 \text {-acamb })_{2}\left(\mathrm{~N}_{2} \mathrm{H}_{4}\right)\right] \cdot \mathrm{H}_{2} \mathrm{O}$, where $\mathrm{M}=\mathrm{Co}$, Cd and $\mathrm{Zn}$; $[\mathrm{M}(3-$ acamb $\left.)_{2}\left(\mathrm{~N}_{2} \mathrm{H}_{4}\right)\right] \cdot \mathrm{H}_{2} \mathrm{O}$, where $\mathrm{M}=\mathrm{Ni}$ and $\mathrm{Co}$ and $\left[\mathrm{M}(3-\mathrm{acamb})_{2}\left(\mathrm{~N}_{2} \mathrm{H}_{4}\right)\right] \cdot 2 \mathrm{H}_{2} \mathrm{O}$ where $\mathrm{M}=\mathrm{Cd}$ and $\mathrm{Zn} ;\left[\mathrm{Co}(4-\mathrm{acamb})_{2}\left(\mathrm{~N}_{2} \mathrm{H}_{4}\right)\right] \cdot \mathrm{H}_{2} \mathrm{O}$ and $\left[\mathrm{M}(4 \text {-acamb })_{2}\left(\mathrm{~N}_{2} \mathrm{H}_{4}\right)\right] \cdot 2 \mathrm{H}_{2} \mathrm{O}$ where $\mathrm{M}=\mathrm{Ni}, \mathrm{Cd}$ and $\mathrm{Zn}, 2$-acambH $=2$-acetamido benzoic acid, 3-acambH = 3-acetamido benzoic acid and 4-acambH = 4-acetamido benzoic acid. Among them Ni, Co and $\mathrm{Cd}$ complexes of 2-acambH and 4-acambH were obtained at pH 5 and 6 respectively, whereas $\mathrm{Zn}$ complexes of both acids were formed at $\mathrm{pH}$ 3. 3-acambH complexes were prepared at pH 5. The IR spectra of the compounds display the $N-N$ stretching frequency absorptions in the range of $984-926 \mathrm{~cm}^{-1}$, which reveals the bridging bidentate coordination of hydrazine. The compounds show $v(\mathrm{C}=\mathrm{O})(\mathrm{asym})$ values in the range 1611-1582 $\mathrm{cm}^{-1}$ and the $v(\mathrm{C}=\mathrm{O})(\mathrm{sym}) \mathrm{values}$ at $1555-1422 \mathrm{~cm}^{-1}$. The difference of $\mathrm{v}(\mathrm{C}=\mathrm{O})$ (asym) and $\mathrm{v}(\mathrm{C}=\mathrm{O})(\mathrm{sym})$, which is found to be $48-162 \mathrm{~cm}^{-1}$, indicates that the carboxylate anion is coordinated to the metal ion in the bidendate fashion. These complexes undergo dehydration in the range of $140-177^{\circ} \mathrm{C}$ first and then oxidative decomposition showing exotherms in the range of $200-278{ }^{\circ} \mathrm{C}$ and in the range of $400-682{ }^{\circ} \mathrm{C}$ to their respective metal oxides. Cadmium and zinc complexes show their intermediates as carbonates. The electronic spectra and the magnetic susceptibility values suggest that the coordination number of the complexes is 6 with distorted octahedral geometry. XRD patterns show isomorphism among the complexes with similar molecular formulae. The SEM-EDS studies reveal the presence of respective metal oxides.
\end{abstract}

Key Words: IR, Simultaneous TG-DTA, Acetamido benzoate, Electronic spectra.

\section{INTRODUCTION}

Hydrazine is the simplest diamine containing two lone pair of electrons. It forms variety of compounds with more number of carboxylic acids, such as simple aliphatic mono and di carboxylic acids ${ }^{1,2}$ aromatic mono and di carboxylic $\operatorname{acids}^{3,4}$ tri and tetra carboxylic acids ${ }^{5,6}$, which have been reported in the literature. Many bis-hydrazine and hydrazinium metal carboxylates are used as precursors for metal oxides and mixed metal oxides ${ }^{7-15}$. Though studies have been done on 4-acetamido benzoate synthesis of lanthanide coordination polymers using 4-acetamido benzoic acid and various modes of coordination of 4-acetamido benzoate (monodentate, chelating and multidentate bridging nature $)^{16-20}$, a detailed study of transition metal complexes of isomeric acetamido benzoic acids in the presence of hydrazine has not been attempted so far. In this paper, we report the synthesis, spectral and thermal studies of some transition metal complexes of 2-acetamido, 3 -acetamido and 4-acetamido benzoates with hydrazine. The structures of the isomeric forms acetamido benzoic acids are given in the Figs. 1-3.

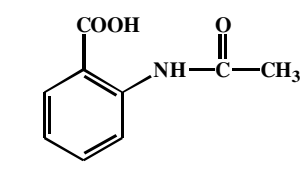
Fig. 1. 2-Acetamido benzoic acid (2-acambH)<smiles>CC(=O)Nc1cccc(C(=O)O)c1</smiles><smiles>CC(=O)Nc1ccc(C(=O)O)cc1</smiles>

Fig. 2. 3-Acetamido benzoic Fig. 3. 4-Acetamido benzoic acid (3-acambH)

\section{EXPERIMENTAL}

The chemicals and solvents used were of AR grade received from Fluka chemicals. The double distilled water was used for the preparation and chemical analyses. Hydrazine hydrate $99.99 \%$ pure was used as such.

Preparation of $\left[\mathrm{M}\{(2-),(3-) \text { and (4-) acamb }\}_{2}\left(\mathbf{N}_{2} \mathbf{H}_{4}\right)\right]$. $\mathrm{xH}_{2} \mathrm{O}$, where 2-acambH =2-acetamido benzoic acid, $\mathrm{M}=$ 
$\mathrm{Ni}$ and $\mathrm{x}=2$ and $\mathrm{M}=\mathrm{Co}, \mathrm{Cd}$ and $\mathrm{x}=1$; where 3-acambH = 3-acetamido benzoic acid, $\mathrm{M}=\mathrm{Ni}, \mathrm{Co}$ and $\mathrm{x}=1$ and $\mathrm{M}=$ $\mathrm{Cd}$ and $\mathrm{x}=2$; where 4-acambH $=4$-acetamido benzoic acid $M=N i, C d$ and $x=2$ and $M=C o$ and $x=1$.

The complexes were prepared by adding an aqueous solution of metal nitrate $\left[\right.$ e.g., $\mathrm{Ni}\left(\mathrm{NO}_{3}\right)_{2} .6 \mathrm{H}_{2} \mathrm{O}, 0.291 \mathrm{~g}, 1 \mathrm{mmol}$ in $20 \mathrm{~mL}$ of $\mathrm{H}_{2} \mathrm{O}$ ] to a clear solution obtained by mixing hydrazine hydrate (99.99\% pure, $0.1 \mathrm{~g}, 2 \mathrm{mmol}$ ) with a slurry of acetamido benzoic acid $(0.1792 \mathrm{~g}, 1 \mathrm{mmol}$ in $60 \mathrm{~mL}$ of hot water). Crystalline products of complexes were formed when the solution mixture was heated over a hot water bath at $80{ }^{\circ} \mathrm{C}$.

2-Acetamido benzoic acid complexes were formed at $\mathrm{pH}$ 5 immediately whereas 3 -acetamido and 4 -acetamido benzoic acid complexes formed at $\mathrm{pH} 5$ when the reaction mixture is heated for $90 \mathrm{~min}$ and at $\mathrm{pH} 6$ when heated for $2 \mathrm{~h} 30 \mathrm{~min}$ respectively.

The complexes formed were filtered through filter paper, washed with distilled water, ethanol then with ether and dried in a desiccator.

Preparation of $\left[\mathrm{Zn}(\mathrm{acamb})_{2}\left(\mathrm{~N}_{2} \mathrm{H}_{4}\right)\right] \cdot \mathrm{xH}_{2} \mathrm{O}$, where 2acambH $=2$-acetamidobenzoic acid and $x=1$ and 3acambH $=3$-acetamido benzoic acid, 4-acambH $=4$ acetamidobenzoic acid and $\mathbf{x}=\mathbf{2}$.

These complexes were also prepared by the same procedure in absolute alcohol medium. As mentioned above, complexes of $\mathbf{2}$ isomer were formed immediately at $\mathrm{pH} 3$ and those of $\mathbf{3}$ and $\mathbf{4}$ isomers were formed at pH 5 and 3 in $90 \mathrm{~min}$ and $2 \mathrm{~h} 30 \mathrm{~min}$ respectively.

The complexes were filtered, washed and dried as mentioned above.

Physico-chemical techniques: The composition was established by chemical analysis. Hydrazine content was determined by titrating against standard $\mathrm{KIO}_{3}$ solution $(0.025$ mol L $\left.{ }^{-1}\right)$ under Andrew's conditions ${ }^{20}$. The metal content was determined by EDTA $\left(0.01 \mathrm{~mol} \mathrm{~L}^{-1}\right)$ complexometric titration ${ }^{20}$ after decomposing a known weight of the sample with $1: 1 \mathrm{HNO}_{3}$. Magnetic measurements were carried out by using Guoy balance and Gauss meter DGM102 (Besto) keeping $\mathrm{Hg}\left[\mathrm{Co}(\mathrm{NCS})_{4}\right]$ as calibrant. The electronic spectra for solid state complexes were obtained using a Varian, Cary 5000 recording spectrophotometer. Infrared spectra were recorded using KBR disc (4000-400 $\left.\mathrm{cm}^{-1}\right)$ on a Shimadzu FTIR-8201 (PC)S spectrophotometer. The simultaneous TG-DTA studies were done on a Perkin Elmer, Diamond TG/DTA analyzer and the curves were obtained using $5-10 \mathrm{mg}$ of the samples at the heating rate of $10^{\circ} \mathrm{C}$ per min in air atmosphere. Platinum cups were employed as sample holders and alumina as reference and the temperature range was ambient to $700{ }^{\circ} \mathrm{C}$. The XRD patterns were recorded on a Bruker AXS $\mathrm{D}_{8}$ advance diffractometer with an X-ray source $\mathrm{Cu}$, wavelength $1.5406 \AA$ using a Si (Li) PSD detector. The elemental analysis was carried out using an Elementar Vario ELIII CHNS elemental analyzer. The SEM with EDS analysis was obtained using JEOL model JSM-6390 LV and JEOL model JED-2300 instrument.

\section{RESULTS AND DISCUSSION}

Electronic spectra and magnetic susceptibility: The absorption maximum and assignments are summarized in Table-1. Since the complexes were insoluble in water and organic solvents, the electronic reflectance spectra were recorded in solid-state. Based on the absorptions term states assigned are ${ }^{4} \mathrm{~T}_{1 \mathrm{~g}}(\mathrm{P}) \rightarrow{ }^{4} \mathrm{~T}_{2 \mathrm{~g}}$ and ${ }^{4} \mathrm{~A}_{2 \mathrm{~g}}$ for cobalt and ${ }^{3} \mathrm{~T}_{2 \mathrm{~g}} \rightarrow$ ${ }^{3} \mathrm{~T}_{1 \mathrm{~g}}$ and ${ }^{1} \mathrm{E}_{\mathrm{g}}$ for nickel complexes. These assignments evidence the distorted octahedral geometry of the complexes ${ }^{21}$. The magnetic moment values obtained for the cobalt and nickel complexes were 5.20 and 3.31 BM respectively, which supports the geometry of cobalt and nickel compounds.

TABLE-1

ELECTRONIC SPECTRA VALUES OF THE COMPLEXES

\begin{tabular}{|c|c|c|}
\hline Complex & Absorption maximum $\left(\mathrm{cm}^{-1}\right)$ & Assignment \\
\hline \multirow{2}{*}{$\begin{array}{l}{\left[\mathrm{Ni}(2 \text {-acamb })_{2} \mathrm{~N}_{2} \mathrm{H}_{4}\right] .} \\
2 \mathrm{H}_{2} \mathrm{O}\end{array}$} & 20534,16807 and 15408 & ${ }^{3} T_{1 g}$ \\
\hline & 8313,7257 and 6725 & ${ }^{3} \mathrm{~T}_{2 \mathrm{~g}}$ \\
\hline \multirow{2}{*}{$\begin{array}{l}{\left[\mathrm{Ni}(3-\mathrm{acamb})_{2}\left(\mathrm{~N}_{2} \mathrm{H}_{4}\right)\right] .} \\
\mathrm{H}_{2} \mathrm{O}\end{array}$} & $\begin{array}{l}20000,16447,13459 \text { and } \\
12136\end{array}$ & ${ }^{3} \mathrm{~T}_{1 \mathrm{~g}}$ \\
\hline & 8224 and 7257 & ${ }^{3} \mathrm{~T}_{2 \mathrm{~g}}$ \\
\hline \multirow{3}{*}{$\begin{array}{l}{\left[\mathrm{Ni}(4-\mathrm{acamb})_{2}\left(\mathrm{~N}_{2} \mathrm{H}_{4}\right)\right] .} \\
2 \mathrm{H}_{2} \mathrm{O}\end{array}$} & 20000 and 13459 & ${ }^{3} \mathrm{~T}_{1 \mathrm{~g}}$ \\
\hline & 11933 & ${ }^{1} \mathrm{E}_{\mathrm{g}}$ \\
\hline & 7955 and 6064 & ${ }^{3} \mathrm{~T}_{2 \mathrm{~g}}$ \\
\hline \multirow{3}{*}{$\begin{array}{l}{\left[\mathrm{Co}(2-\mathrm{acamb})_{2}\left(\mathrm{~N}_{2} \mathrm{H}_{4}\right)\right] .} \\
\mathrm{H}_{2} \mathrm{O}\end{array}$} & 21186 & ${ }^{4} \mathrm{~T}_{1 \mathrm{~g}}(\mathrm{P})$ \\
\hline & 11806 & ${ }^{4} \mathrm{~A}_{2 g}$ \\
\hline & 8467,7348 and 6667 & ${ }^{4} \mathrm{~T}_{2 \mathrm{~g}}$ \\
\hline \multirow{3}{*}{$\begin{array}{l}{\left[\mathrm{Co}(3-\mathrm{acamb})_{2}\left(\mathrm{~N}_{2} \mathrm{H}_{4}\right)\right] .} \\
\mathrm{H}_{2} \mathrm{O}\end{array}$} & 23148 & ${ }^{4} \mathrm{~T}_{1 \mathrm{~g}}(\mathrm{P})$ \\
\hline & 11561 & ${ }^{4} \mathrm{~A}_{2 \mathrm{~g}}$ \\
\hline & $8313,7117,6489$ and 5780 & ${ }^{4} \mathrm{~T}_{2 \mathrm{~g}}$ \\
\hline$\left[\mathrm{Co}(4 \text {-acamb })_{2}\left(\mathrm{~N}_{2} \mathrm{H}_{4}\right)\right]$. & 12755 & ${ }^{4} \mathrm{~A}_{2 \mathrm{~g}}$ \\
\hline $\mathrm{H}_{2} \mathrm{O}$ & $8224,7184,6667$ and 6017 & ${ }^{4} \mathrm{~T}_{2 \mathrm{~g}}$ \\
\hline
\end{tabular}

IR spectra of complexes: The IR spectral data of the complexes are summarized in Table-2. The pka values of 2-acambH, 3-acambH and 4-acambH were found to be 3.63, 4.07 and 4.28 respectively. These indicate that 4 -isomer is least acidic and the lowest value of $\mathrm{v}(\mathrm{C}=\mathrm{O}) 1672 \mathrm{~cm}^{-1}$ of the same may be due to its highest pka value.

The IR spectra of pure acids show absorption at 1707 , 1694 and $1672 \mathrm{~cm}^{-1}$ corresponding to $\mathrm{v}(\mathrm{C}=\mathrm{O}$ ) (acid). But the spectra of complexes show $\mathrm{v}(\mathrm{C}=\mathrm{O})$ asym (acid) at 1582-1611 $\mathrm{cm}^{-1}$ and $v(\mathrm{C}=\mathrm{O})$ sym (acid) in the range $1555-1422 \mathrm{~cm}^{-1}$ with the difference of $48-162 \mathrm{~cm}^{-1}$ between $\mathrm{v}(\mathrm{C}=\mathrm{O})$ (asym) and $v(\mathrm{C}=\mathrm{O})$ (sym), which supports the bidental coordination of carboxylate ions to metal. The absorption at $984-926 \mathrm{~cm}^{-1}$ observed in IR spectra of complexes is assigned to $v(\mathrm{~N}-\mathrm{N})$ stretching of hydrazine present in the complexes, which reveals that $\mathrm{N}_{2} \mathrm{H}_{4}$ is coordinated to metal ion in bridged bidentate fashion. The $\mathrm{O}-\mathrm{H}$ stretch of water molecules are noticed at $3543-3304 \mathrm{~cm}^{-1}$ in all complexes. An additional band observed at $594-518 \mathrm{~cm}^{-1}$ also supports the presence of lattice water molecules ${ }^{22}$. The $\mathrm{C}=\mathrm{O}$ frequency of amide group of the compounds is observed at $1709-1635 \mathrm{~cm}^{-1}$. The N-H stretching frequencies of amide and that of hydrazine are found to be a merged broad band at $3281-3173 \mathrm{~cm}^{-1}$.

Thermal data of all the complexes are given in Table- 3 . Thermal data of $\left[\mathrm{M}(2 \text {-acamb })_{2}\left(\mathrm{~N}_{2} \mathrm{H}_{4}\right)_{2}\right] \cdot \mathbf{x H}_{2} \mathrm{O}$ complexes, where $M=N i$ and $x=2$, where $M=C o, C d$ and $\mathrm{Zn}$ and $x=1$

The 2-acambH complexes show endothermic dehydration in the range $140-177^{\circ} \mathrm{C}$. The high temperature dehydration reveals that these lattice water molecules are held up strongly ${ }^{22}$. $\mathrm{Co}$ and $\mathrm{Cd}$ complexes show exothermic dehydrazi-nation at $214-275^{\circ} \mathrm{C}$, whereas the dehydrazination was not clearly visible in $\mathrm{Ni}$ and $\mathrm{Zn}$ compounds. 
ABLE-2

ANALYTICAL AND IR DATA OF COMPLEXES

\begin{tabular}{|c|c|c|c|c|c|c|c|c|c|c|c|c|c|}
\hline \multirow[b]{2}{*}{ m.f. } & \multicolumn{5}{|c|}{ Analytical data } & \multicolumn{8}{|c|}{ IR data $\left(\mathrm{cm}^{-1}\right) \mathrm{b}=$ broad; $\mathrm{s}=\operatorname{sharp} ; \mathrm{m}=$ medium } \\
\hline & $\begin{array}{c}\text { C } \\
\text { Found } \\
\text { (calc.) }\end{array}$ & $\begin{array}{c}\text { H } \\
\text { Found } \\
\text { (calc.) }\end{array}$ & $\begin{array}{c}\mathrm{N} \\
\text { Found } \\
\text { (calc.) }\end{array}$ & $\begin{array}{l}\mathrm{N}_{2} \mathrm{H}_{4} \\
\text { Found } \\
\text { (calc.) }\end{array}$ & $\begin{array}{c}\text { M } \\
\text { Found } \\
\text { (calc.) }\end{array}$ & $\begin{array}{c}v \\
(\mathrm{C}=\mathrm{O}) \\
\text { asym }\end{array}$ & $\begin{array}{c}v \\
(\mathrm{C}=\mathrm{O}) \\
\text { sym }\end{array}$ & $\begin{array}{c}v \\
(\mathrm{~N}-\mathrm{N})\end{array}$ & $\begin{array}{c}v \\
(\mathrm{OH})\end{array}$ & $\begin{array}{c}v \\
(\mathrm{~N}-\mathrm{H})\end{array}$ & $\begin{array}{c}v(\mathrm{C}=\mathrm{O}) \\
\text { (amido } \\
\mathrm{gp})\end{array}$ & $\begin{array}{c}\rho_{\mathrm{r}} \\
\mathrm{H}_{2} \mathrm{O}\end{array}$ & $\begin{array}{c}v \\
(\mathrm{M}-\mathrm{O})\end{array}$ \\
\hline \multicolumn{14}{|c|}{ 2-acambH complexes } \\
\hline $\begin{array}{l}{\left[\mathrm{Ni}(2 \text {-acamb })_{2}\right.} \\
\left.\mathrm{N}_{2} \mathrm{H}_{4}\right] \cdot 2 \mathrm{H}_{2} \mathrm{O}\end{array}$ & $45.6(44.7)$ & $4.4(5.0)$ & $12.0(11.6)$ & $6.4(6.6)$ & $12.9(12.2)$ & $1595 \mathrm{~b}$ & $1433 \mathrm{~b}$ & $964 \mathrm{~s}$ & $3464 b$ & $3233 \mathrm{~b}$ & $1684 \mathrm{~m}$ & $527 \mathrm{~s}$ & $422 \mathrm{~m}$ \\
\hline $\begin{array}{l}{\left[\mathrm{Co}\left(2-\mathrm{acamb}_{2}\right.\right.} \\
\left.\left(\mathrm{N}_{2} \mathrm{H}_{4}\right)\right] \cdot \mathrm{H}_{2} \mathrm{O}\end{array}$ & $46.0(46.4)$ & $4.3(4.8)$ & 12.1(12.0) & $6.4(6.9)$ & $12.4(12.7)$ & $1589 \mathrm{~s}$ & $1439 \mathrm{~s}$ & $966 \mathrm{~s}$ & $3304 \mathrm{~m}$ & $3175 \mathrm{~m}$ & $1661 \mathrm{~s}$ & $594 \mathrm{~s}$ & $438 \mathrm{~s}$ \\
\hline $\begin{array}{l}{\left[\mathrm{Cd}(2 \text {-acamb })_{2}\right.} \\
\left.\left(\mathrm{N}_{2} \mathrm{H}_{4}\right)\right] \cdot \mathrm{H}_{2} \mathrm{O}\end{array}$ & $41.8(41.6)$ & $4.3(4.3)$ & $10.7(10.8)$ & $6.3(6.2)$ & 21.3(21.7) & $1584 \mathrm{~b}$ & $1422 \mathrm{~b}$ & $959 \mathrm{~s}$ & $3543 \mathrm{~s}$ & $3235 \mathrm{~b}$ & $1676 \mathrm{~m}$ & $594 \mathrm{~s}$ & $509 \mathrm{~s}$ \\
\hline $\begin{array}{l}{\left[\mathrm{Zn}(2 \text {-acamb })_{2}\right.} \\
\left.\left(\mathrm{N}_{2} \mathrm{H}_{4}\right)\right] \cdot \mathrm{H}_{2} \mathrm{O}\end{array}$ & $45.3(45.8)$ & $4.5(4.7)$ & $11.8(11.9)$ & $6.8(6.8)$ & 13.7(13.9) & $1599 \mathrm{~s}$ & $1447 \mathrm{~s}$ & $970 \mathrm{~s}$ & $3320 \mathrm{~s}$ & $3219 b$ & $1661 \mathrm{~s}$ & $567 \mathrm{~s}$ & $463 \mathrm{~s}$ \\
\hline \multicolumn{14}{|c|}{ 3-acambH complexes } \\
\hline $\begin{array}{l}\mathrm{Ni}(3 \text {-acamb })_{2} \\
\left.\left(\mathrm{~N}_{2} \mathrm{H}_{4}\right)\right] \cdot \mathrm{H}_{2} \mathrm{O}\end{array}$ & $46.5(46.4)$ & $4.5(4.8)$ & $12.0(12.0)$ & $6.7(6.9)$ & $12.4(12.6)$ & $1582 \mathrm{~s}$ & $1460 \mathrm{~s}$ & $984 \mathrm{~s}$ & $3392 b$ & $3227 b$ & $1709 \mathrm{~b}$ & $525 \mathrm{~s}$ & $476 \mathrm{~m}$ \\
\hline $\begin{array}{l}{\left[\mathrm{Co}(3 \text {-acamb })_{2}\right.} \\
\left.\left(\mathrm{N}_{2} \mathrm{H}_{4}\right)\right] \cdot \mathrm{H}_{2} \mathrm{O}\end{array}$ & $46.4(46.4)$ & $4.7(4.8)$ & $12.1(12.0)$ & $6.9(6.9)$ & $(12.6(12.7)$ & $1611 \mathrm{~m}$ & $1487 \mathrm{~s}$ & $980 \mathrm{~s}$ & $3339 \mathrm{~s}$ & $3271 \mathrm{~b}$ & $1635 \mathrm{~b}$ & $552 \mathrm{~s}$ & $459 \mathrm{~s}$ \\
\hline $\begin{array}{l}{\left[\mathrm{Cd}(3 \text {-acamb })_{2}\right.} \\
\left.\left(\mathrm{N}_{2} \mathrm{H}_{4}\right)\right] \cdot 2 \mathrm{H}_{2} \mathrm{O}\end{array}$ & $41.3(40.2)$ & $4.2(4.5)$ & $10.9(10.4)$ & $6.5(6.0)$ & 20.1(20.9) & $1611 \mathrm{~s}$ & $1462 \mathrm{~s}$ & $926 \mathrm{~m}$ & $3376 \mathrm{~b}$ & $3281 \mathrm{~s}$ & $1659 \mathrm{~s}$ & $559 \mathrm{~s}$ & $459 \mathrm{~s}$ \\
\hline $\begin{array}{l}{\left[\mathrm{Zn}(3 \text {-acamb })_{2}\right.} \\
\left.\left(\mathrm{N}_{2} \mathrm{H}_{4}\right)\right] \cdot 2 \mathrm{H}_{2} \mathrm{O} \\
\end{array}$ & $43.9(44.1)$ & $4.7(4.9)$ & $11.6(11.4)$ & $7.0(6.5)$ & $14.3(13.4)$ & $1607 \mathrm{~s}$ & $1487 \mathrm{~s}$ & $974 \mathrm{~s}$ & $3305 \mathrm{~s}$ & $3215 b$ & $1607 \mathrm{~b}$ & $559 \mathrm{~s}$ & $430 \mathrm{~s}$ \\
\hline \multicolumn{14}{|c|}{ 4-acambH complexes } \\
\hline $\begin{array}{l}\mathrm{Ni}(4-\mathrm{acamb})_{2} \\
\left.\left(\mathrm{~N}_{2} \mathrm{H}_{4}\right)\right] \cdot 2 \mathrm{H}_{2} \mathrm{O}\end{array}$ & $44.4(44.7)$ & $4.5(5.0)$ & $11.8(11.6)$ & $7.4(6.6)$ & $13.2(13.1)$ & $1609 \mathrm{~b}$ & $1530 \mathrm{~b}$ & $964 \mathrm{~s}$ & $3360 \mathrm{~m}$ & $3183 \mathrm{~b}$ & $1672 \mathrm{~m}$ & $544 \mathrm{~s}$ & $500 \mathrm{~s}$ \\
\hline $\begin{array}{l}{\left[\mathrm{Co}(4-\mathrm{acamb})_{2}\right.} \\
\left.\left(\mathrm{N}_{2} \mathrm{H}_{4}\right)\right] \cdot \mathrm{H}_{2} \mathrm{O}\end{array}$ & $46.2(46.4)$ & $4.7(4.8)$ & $12.0(12.0)$ & $6.7(6.9)$ & $12.8(12.7)$ & $1601 \mathrm{~b}$ & $1528 \mathrm{~b}$ & $968 \mathrm{~s}$ & $3335 \mathrm{~b}$ & $3173 b$ & $1680 \mathrm{~b}$ & $546 \mathrm{~s}$ & $500 \mathrm{~s}$ \\
\hline $\begin{array}{l}{\left[\mathrm{Cd}(4-\mathrm{acamb})_{2}\right.} \\
\left.\left(\mathrm{N}_{2} \mathrm{H}_{4}\right)\right] \cdot 2 \mathrm{H}_{2} \mathrm{O}\end{array}$ & $41.2(40.2)$ & $3.8(4.5)$ & $11.3(10.4)$ & $6.2(6.0)$ & 21.3(20.9) & $1603 \mathrm{~b}$ & $1555 \mathrm{~b}$ & $964 \mathrm{~s}$ & $3377 \mathrm{~b}$ & $3260 \mathrm{~b}$ & $1668 \mathrm{~b}$ & $581 \mathrm{~s}$ & $500 \mathrm{~s}$ \\
\hline $\begin{array}{l}{\left[\mathrm{Zn}(4-\mathrm{acamb})_{2}\right.} \\
\left.\left(\mathrm{N}_{2} \mathrm{H}_{4}\right) \cdot 2 \mathrm{H}_{2} \mathrm{O}\right]\end{array}$ & $44.7(44.1)$ & 4.1(4.9) & $12.4(11.4)$ & $7.0(6.5)$ & $14.4(13.4)$ & $1597 \mathrm{~s}$ & $1521 \mathrm{~s}$ & $972 \mathrm{~s}$ & $3315 \mathrm{~s}$ & $3270 \mathrm{~b}$ & $1672 \mathrm{~m}$ & $518 \mathrm{~s}$ & $503 \mathrm{~s}$ \\
\hline
\end{tabular}

The cadmium complex shows a broad exotherm at $400{ }^{\circ} \mathrm{C}$ indicating the formation of metal carbonate intermediate ${ }^{23,24}$ and the corresponding weight loss is observed between 300 $470{ }^{\circ} \mathrm{C}$ in its thermogravimetric curve. It is also inferred that the complex undergoes oxidative decomposition to form the metal oxide residue, which is accompanied by an exothermic doublet at 490 and $556^{\circ} \mathrm{C}$ indicating the weight loss temperature of $470-700^{\circ} \mathrm{C}$.

The TG-DTA curves of Ni, Co and $\mathrm{Zn}$ complexes show that the dehydrazinated metal carboxylates decompose to give the metal oxide as their final residue. As the intermediates are highly unstable and they could not be isolated. They show exotherms in the range $420-500{ }^{\circ} \mathrm{C}$ attributing the formation of metal oxide residue with the mass loss temperature in the range of $200-700{ }^{\circ} \mathrm{C}$ is observed in their TG.

\section{Reaction Scheme-I} below:

The reaction scheme of the 2-acambH complexes is given

$$
\begin{gathered}
{\left[\mathrm{M}\left\{\mathrm{C}_{6} \mathrm{H}_{4}\left(\mathrm{CH}_{3} \mathrm{CONH}\right) \mathrm{COO}\right\}_{2}\left(\mathrm{~N}_{2} \mathrm{H}_{4}\right)\right] \cdot \mathrm{xH}_{2} \mathrm{O} \frac{140-177^{\circ} \mathrm{C}}{-\mathrm{H}_{2} \mathrm{O}}} \\
{\left[\mathrm{M}\left\{\mathrm{C}_{6} \mathrm{H}_{4}\left(\mathrm{CH}_{3} \mathrm{CONH}\right) \mathrm{COO}\right\}_{2}\left(\mathrm{~N}_{2} \mathrm{H}_{4}\right)\right]}
\end{gathered}
$$

where, $\mathrm{M}=\mathrm{Ni}, \mathrm{Co}, \mathrm{Cd}$ and $\mathrm{Zn}$

$$
\begin{array}{r}
{\left[\mathrm{M}\left\{\mathrm{C}_{6} \mathrm{H}_{4}\left(\mathrm{CH}_{3} \mathrm{CONH}\right) \mathrm{COO}\right\}_{2}\left(\mathrm{~N}_{2} \mathrm{H}_{4}\right)\right] \frac{214-275^{\circ} \mathrm{C}}{-\mathrm{N}_{2} \mathrm{H}_{4}}} \\
\mathrm{M}\left\{\mathrm{C}_{6} \mathrm{H}_{4}\left(\mathrm{CH}_{3} \mathrm{CONH}\right) \mathrm{COO}\right\}_{2}
\end{array}
$$

where, $\mathrm{M}=\mathrm{Co}$ and $\mathrm{Cd}$

$$
\mathrm{M}\left\{\mathrm{C}_{6} \mathrm{H}_{4}\left(\mathrm{CH}_{3} \mathrm{CONH}\right) \mathrm{COO}\right\}_{2} \stackrel{420-500{ }^{\circ} \mathrm{C}}{\longrightarrow} \mathrm{MO}
$$

where, $\mathrm{M}=\mathrm{Ni}, \mathrm{Co}$ and $\mathrm{Zn}$ and $\left.\mathrm{CoO}=\mathrm{Co}_{3} \mathrm{O}_{4}\right)$

$$
\left[\mathrm{Cd}\left\{\mathrm{C}_{6} \mathrm{H}_{4}\left(\mathrm{CH}_{3} \mathrm{CONH}\right) \mathrm{COO}\right\}_{2}\right] \stackrel{40{ }^{\circ} \mathrm{C}}{\stackrel{490-556^{\circ} \mathrm{C}}{\longrightarrow}} \mathrm{CdCO}_{3}
$$

Thermal data of $\left[\mathrm{M}(3 \text {-acamb })_{2}\left(\mathrm{~N}_{2} \mathrm{H}_{4}\right)_{2}\right] \cdot \mathrm{xH}_{2} \mathrm{O}$; where $\mathrm{M}=$ $\mathrm{Ni}, \mathrm{Co}$ and $\mathrm{x}=1 ; \mathrm{M}=\mathrm{Cd}, \mathrm{Zn}$ and $\mathrm{x}=2$

Similar to 2-acambH complexes, 3-acambH complexes show an endothermic dehydration at $122-160{ }^{\circ} \mathrm{C}$. Ni and $\mathrm{Co}$ complexes show exothermic dehydrazination in the range of 230-260 ${ }^{\circ} \mathrm{C}$, while $\mathrm{Cd}$ and $\mathrm{Zn}$ complexes do not. The intermediates of $\mathrm{Ni}, \mathrm{Co}$ and $\mathrm{Zn}$ compounds were unstable and could not be identified. They further decompose to their respective metal oxides at $500-600{ }^{\circ} \mathrm{C}$. In case of cadmium oxidative decomposition occurs via its carbonate intermediate. Its decomposition temperature, $494^{\circ} \mathrm{C}$ was comparable to that of the reported ${ }^{6}$, with the corresponding weight loss between 400 $700^{\circ} \mathrm{C}$.

\section{Reaction Scheme-II}

The reaction scheme of 3-acetamidobenzoate complexes is given as follows:

$$
\begin{gathered}
{\left[\mathrm{M}\left\{\mathrm{C}_{6} \mathrm{H}_{4}\left(\mathrm{CH}_{3} \mathrm{CONH}\right) \mathrm{COO}\right\}_{2}\left(\mathrm{~N}_{2} \mathrm{H}_{4}\right)\right] \cdot \times \mathrm{H}_{2} \mathrm{O} \frac{122-160{ }^{\circ} \mathrm{C}}{-\mathrm{H}_{2} \mathrm{O}}} \\
{\left[\mathrm{M}\left\{\mathrm{C}_{6} \mathrm{H}_{4}\left(\mathrm{CH}_{3} \mathrm{CONH}\right) \mathrm{COO}\right\}_{2}\left(\mathrm{~N}_{2} \mathrm{H}_{4}\right)\right]}
\end{gathered}
$$

where, $\mathrm{M}=\mathrm{Ni}, \mathrm{Co}, \mathrm{Cd}$ and $\mathrm{Zn}$

$\left[\mathrm{M}\left\{\mathrm{C}_{6} \mathrm{H}_{4}\left(\mathrm{CH}_{3} \mathrm{CONH}\right) \mathrm{COO}\right\}_{2}\left(\mathrm{~N}_{2} \mathrm{H}_{4}\right)\right] \stackrel{230-260{ }^{\circ} \mathrm{C}}{\underset{-\mathrm{N}_{2} \mathrm{H}_{4}}{\longrightarrow}}$

where, $\mathrm{M}=\mathrm{Ni}$ and $\mathrm{Co}$

$\left[\mathrm{M}\left\{\mathrm{C}_{6} \mathrm{H}_{4}\left(\mathrm{CH}_{3} \mathrm{CONH}\right) \mathrm{COO}\right\}_{2}\right]$

$$
\left.\left[\mathrm{M}\left\{\mathrm{C}_{6} \mathrm{H}_{4}\left(\mathrm{CH}_{3} \mathrm{CONH}\right) \mathrm{COO}\right\}_{2}\right)\right] \stackrel{446-600{ }^{\circ} \mathrm{C}}{\longrightarrow}[\mathrm{MO}]
$$

where, $\mathrm{M}=\mathrm{Ni}, \mathrm{Co}$, and $\mathrm{Zn}$ and $\mathrm{CoO}=\mathrm{Co}_{3} \mathrm{O}_{4}$

$$
\left.\left[\mathrm{Cd}\left\{\mathrm{C}_{6} \mathrm{H}_{4}\left(\mathrm{CH}_{3} \mathrm{CONH}\right) \mathrm{COO}\right\}_{2}\right)\right] \stackrel{210-317^{\circ} \mathrm{C}}{\longrightarrow} \mathrm{CdCO}_{3}
$$

Thermal data of $\left[\mathrm{M}(4 \text {-acamb })_{2}\left(\mathrm{~N}_{2} \mathrm{H}_{4}\right)_{2}\right] \cdot \mathrm{xH}_{2} \mathrm{O}$; where $\mathrm{M}=$ $\mathrm{Ni}, \mathrm{Cd}, \mathrm{Zn}$ and $\mathrm{x}=2 ; \mathrm{M}=\mathrm{Co}$ and $\mathrm{x}=1$ 


\begin{tabular}{|c|c|c|c|c|c|}
\hline \multicolumn{6}{|c|}{$\begin{array}{c}\text { TABLE-3 } \\
\text { THERMAL DATA OF COMPLEXES }\end{array}$} \\
\hline \multirow{3}{*}{ Complex } & \multirow{3}{*}{ DTA Temp. $\left({ }^{\circ} \mathrm{C}\right)$} & \multicolumn{3}{|c|}{ Thermogravimetry } & \multirow{3}{*}{ Nature of the reaction } \\
\hline & & \multirow{2}{*}{ Temp. Range } & \multicolumn{2}{|c|}{ Weight Loss (\%) } & \\
\hline & & & Obsd. & Calcd. & \\
\hline \multirow{3}{*}[\mathrm{Ni}(2-\mathrm{acamb})_{2}\mathrm{N}_{2}\mathrm{H}_{4}]{$\cdot 2 \mathrm{H}_{2} \mathrm{O}$} & $150(+)$ & $60-200$ & 7.1 & 7.2 & Dehydration \\
\hline & $278(-)$ & & & & \\
\hline & $420(-)$ & $200-700$ & 84.3 & 84.5 & Formation of nickel oxide \\
\hline \multirow{3}{*}{$\mathrm{Co}\left(2-\mathrm{acamb}_{2}\left(\mathrm{~N}_{2} \mathrm{H}_{4}\right)\right] \cdot \mathrm{H}_{2} \mathrm{O}$} & $177(+)$ & $60-190$ & 4.0 & 3.9 & Dehydration \\
\hline & 214(-) & $190-300$ & 10.5 & 10.7 & Dehydrazination \\
\hline & $400(-)$ & $300-700$ & 82.6 & 82.8 & Formation of cobalt oxide $\left(\mathrm{Co}_{3} \mathrm{O}_{4}\right)$ \\
\hline \multirow{4}{*}[\mathrm{Cd}(2-\mathrm{acamb})_{2}(\mathrm{N}_{2}\mathrm{H}_{4})]{$\cdot \mathrm{H}_{2} \mathrm{O}$} & $160(+)$ & $40-180$ & 3.6 & 3.5 & Dehydration \\
\hline & $275(-)$ & $180-300$ & 9.8 & 9.6 & Dehydrazination \\
\hline & $400(-)$ & $300-480$ & 57.1 & 57.1 & Formation of cadmium carbonate \\
\hline & $490(-)$ & $480-700$ & 75.0 & 75.2 & Formation of cadmium oxide \\
\hline \multirow[t]{2}{*}[\mathrm{Zn}(2\text{-acamb})_{2}(\mathrm{N}_{2}\mathrm{H}_{4})]{$\cdot \mathrm{H}_{2} \mathrm{O}$} & $274(-)$ & $180-700$ & 83.0 & 82.7 & Formation of zinc oxide \\
\hline & $500(-)$ & & & & \\
\hline \multirow{4}{*}[\mathrm{Ni}(3\text{-acamb})_{2}(\mathrm{N}_{2}\mathrm{H}_{4})]{$\cdot \mathrm{H}_{2} \mathrm{O}$} & $140(+)$ & $100-200$ & 3.6 & 3.9 & Dehydration \\
\hline & $260(-)$ & $200-400$ & 10.7 & 10.8 & Dehydrazination \\
\hline & $500(-)$ & & & & \\
\hline & $590(-)$ & $4000-700$ & 84.7 & 84.9 & Formation of nickel oxide \\
\hline \multirow{4}{*}[\mathrm{Co}(3\text{-acamb})_{2}(\mathrm{N}_{2}\mathrm{H}_{4})]{$\cdot \mathrm{H}_{2} \mathrm{O}$} & $160(+)$ & $120-200$ & 3.8 & 3.9 & Dehydration \\
\hline & $230(-)$ & $200-340$ & 10.5 & 10.7 & Dehydrazination \\
\hline & $446(-)$ & & & & \\
\hline & $\begin{array}{l}540(-) \\
600(-)\end{array}$ & $340-700$ & 83.8 & 83.9 & Formation of cobalt oxide \\
\hline \multirow[b]{3}{*}[\mathrm{Cd}(3-\mathrm{acamb})_{2}(\mathrm{N}_{2}\mathrm{H}_{4})]{$\cdot 2 \mathrm{H}_{2} \mathrm{O}$} & $122(+)$ & $80-200$ & 6.6 & 6.7 & Dehydration \\
\hline & $210(-)$ & $200-400$ & 55.2 & 55.0 & Formation of cadmium carbonate \\
\hline & $317(-)$ & & & & \\
\hline \multirow{4}{*}[\mathrm{Zn}(3-\mathrm{acamb})_{2}(\mathrm{N}_{2}\mathrm{H}_{4})]{$\cdot 2 \mathrm{H}_{2} \mathrm{O}$} & $145(+)$ & $100-200$ & 7.2 & 7.5 & Dehydration \\
\hline & $240(-)$ & & & & \\
\hline & $468(-)$ & $200-700$ & 83.4 & 83.3 & Formation of zinc oxide \\
\hline & $553(-)$ & & & & \\
\hline \multirow{4}{*}[\mathrm{Ni}(4-\mathrm{acamb})_{2}(\mathrm{N}_{2}\mathrm{H}_{4})]{$\cdot 2 \mathrm{H}_{2} \mathrm{O}$} & $175(+)$ & $50-190$ & 7.4 & 7.5 & Dehydration \\
\hline & $251(-)$ & $190-390$ & 14.0 & 14.1 & Dehydrazination \\
\hline & $401(-)$ & $390-700$ & 85.3 & 85.5 & Formation of nickel oxide \\
\hline & $486(-)\}$ & & & & \\
\hline \multirow{5}{*}[\mathrm{Co}(4-\mathrm{acamb})_{2}(\mathrm{N}_{2}\mathrm{H}_{4})]{$\cdot \mathrm{H}_{2} \mathrm{O}$} & $120(+)$ & $60-180$ & 4.0 & 3.9 & Dehydration \\
\hline & $239(-)$ & $180-280$ & 10.6 & 10.7 & Dehydrazination \\
\hline & $324(-) 7$ & & & & \\
\hline & $390(-)\}$ & & & & \\
\hline & $450(-)$ & $280-700$ & 82.6 & 82.8 & Formation of cobalt oxide $\left(\mathrm{Co}_{3} \mathrm{O}_{4}\right)$ \\
\hline \multirow{5}{*}[\mathrm{Cd}(4-\mathrm{acamb})_{2}(\mathrm{N}_{2}\mathrm{H}_{4})]{$\cdot 2 \mathrm{H}_{2} \mathrm{O}$} & $150(+)$ & $50-180$ & 6.8 & 6.7 & Dehydration \\
\hline & $240(-)$ & $180-320$ & 12.9 & 12.7 & Dehydrazination \\
\hline & $401(-)$ & $320-460$ & 55.0 & 55.2 & Formation of cadmium carbonate \\
\hline & $625(-)\}$ & $460-800$ & 75.8 & 76.0 & \\
\hline & $682(-) J$ & & & & \\
\hline \multirow{4}{*}[\mathrm{Zn}(4-\mathrm{acamb})_{2}(\mathrm{N}_{2}\mathrm{H}_{4})]{$\cdot 2 \mathrm{H}_{2} \mathrm{O}$} & $145(+)$ & $75-160$ & 7.2 & 7.4 & Dehydration \\
\hline & $236(-)\}$ & & & & \\
\hline & $350(-)\}$ & $160-400$ & 63.8 & 64.0 & Formation of zinc carbonate \\
\hline & $533(+)$ & $400-600$ & 83.9 & 84.0 & Formation of zinc oxide \\
\hline
\end{tabular}

4-acambH complexes show endothermic dehydration at $120-175^{\circ} \mathrm{C}$. Exothermic dehydrazination is observed between $239-251^{\circ} \mathrm{C}$ for $\mathrm{Ni}, \mathrm{Co}$ and $\mathrm{Cd}$ complexes and no distinct step was observed in case of $\mathrm{Zn}$ complex.

$\mathrm{Ni}$ and Co complexes undergo oxidative decomposition to their metal oxide as final residue accompanied by the exotherms in the range $324-486{ }^{\circ} \mathrm{C}$ with a corresponding weight loss between $230-700^{\circ} \mathrm{C}$.

In the final step, cadmium and zinc show exothermic decomposition at 350 and $401^{\circ} \mathrm{C}$ via the carbonate interme- diate $^{5,6}$, which decompose further to their metal oxides showing broad exotherms in the range of $533-682^{\circ} \mathrm{C}$ showing the mass loss between $400-800{ }^{\circ} \mathrm{C}$, to $75.8 \%$ for cadmium and $83.9 \%$ for zinc.

The TG-DTA of $\left[\mathrm{Ni}(2 \text {-acamb })_{2}\left(\mathrm{~N}_{2} \mathrm{H}_{4}\right)\right] \cdot 2 \mathrm{H}_{2} \mathrm{O},[\mathrm{Cd}(3-$ acamb $\left.)_{2}\left(\mathrm{~N}_{2} \mathrm{H}_{4}\right)\right] \cdot 2 \mathrm{H}_{2} \mathrm{O}$ and $\left[\mathrm{Zn}(4 \text {-acamb })_{2}\left(\mathrm{~N}_{2} \mathrm{H}_{4}\right)\right] .2 \mathrm{H}_{2} \mathrm{O}$ complexes are shown as representative examples in Figs. 4-6.

\section{Reaction Scheme-III}

The reaction scheme of 4-acetamidobenzoate complexes are given as follows: 


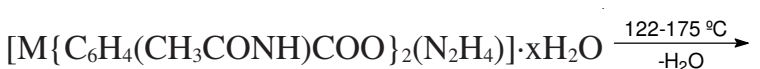
$\left[\mathrm{M}\left\{\mathrm{C}_{6} \mathrm{H}_{4}\left(\mathrm{CH}_{3} \mathrm{CONH}\right) \mathrm{COO}\right\}_{2}\left(\mathrm{~N}_{2} \mathrm{H}_{4}\right)\right]$ where, $\mathrm{M}=\mathrm{Ni}$, $\mathrm{Co}, \mathrm{Cd}$ and $\mathrm{Zn}$

$$
\begin{array}{r}
{\left[\mathrm{M}\left\{\mathrm{C}_{6} \mathrm{H}_{4}\left(\mathrm{CH}_{3} \mathrm{CONH}\right) \mathrm{COO}\right\}_{2}\left(\mathrm{~N}_{2} \mathrm{H}_{4}\right)\right] \stackrel{239-251{ }^{\circ} \mathrm{C}}{-\mathrm{N}_{2} \mathrm{H}_{4}}} \\
{\left[\mathrm{M}\left\{\mathrm{C}_{6} \mathrm{H}_{4}\left(\mathrm{CH}_{3} \mathrm{CONH}\right) \mathrm{COO}\right\}_{2}\right]}
\end{array}
$$

where, $\mathrm{M}=\mathrm{Ni}$ and $\mathrm{Co}$

$$
\left[\mathrm{M}\left\{\mathrm{C}_{6} \mathrm{H}_{4}\left(\mathrm{CH}_{3} \mathrm{CONH}\right) \mathrm{COO}\right\}_{2}\right] \stackrel{324-486{ }^{\circ} \mathrm{C}}{\longrightarrow}[\mathrm{MO}]
$$

where, $\mathrm{M}=\mathrm{Ni}$ and $\mathrm{Co}$ and $\mathrm{CoO}=\mathrm{Co}_{3} \mathrm{O}_{4}$

$\left.\left[\mathrm{Cd}\left\{\mathrm{C}_{6} \mathrm{H}_{4}\left(\mathrm{CH}_{3} \mathrm{CONH}\right) \mathrm{COO}\right\}_{2}\right)\right] \stackrel{201{ }^{\circ} \mathrm{C}}{\longrightarrow} \mathrm{CdCO}_{3}$
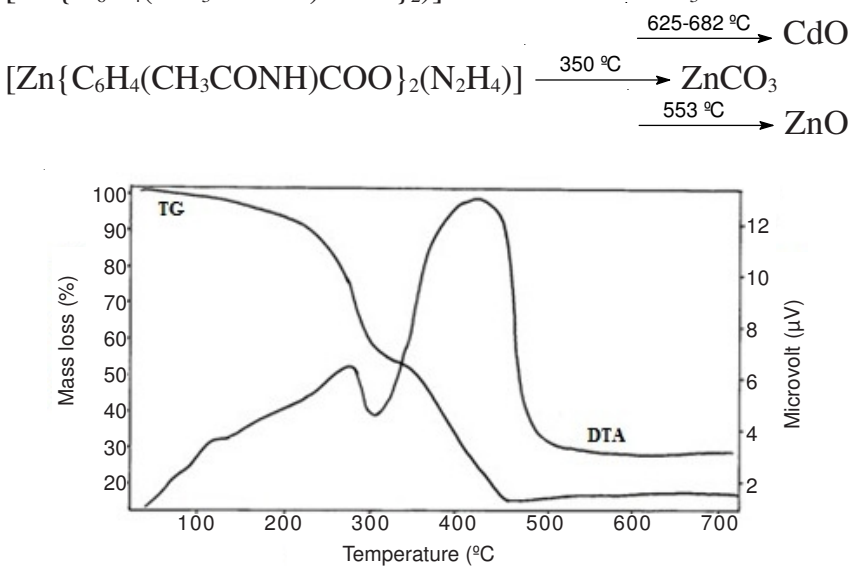

Fig. 4. TG-DTA of $\left[\mathrm{Ni}(2 \text {-acamb })_{2} \mathrm{~N}_{2} \mathrm{H}_{4} \cdot 2 \mathrm{H}_{2} \mathrm{O}\right.$

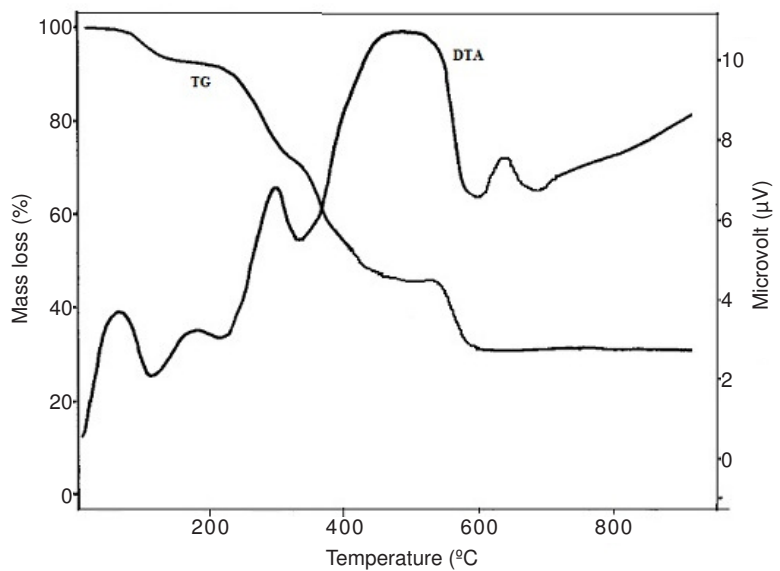

Fig. 5. TG-DTA of $\left[\mathrm{Cd}(2 \text {-acamb })_{2} \mathrm{~N}_{2} \mathrm{H}_{4} \cdot 2 \mathrm{H}_{2} \mathrm{O}\right.$

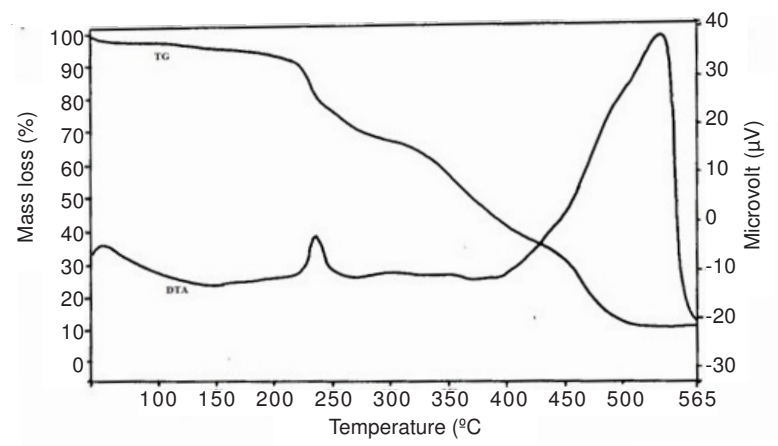

Fig. 6. TG-DTA of $\left[\mathrm{Zn}(4-\mathrm{acamb})_{2}\left(\mathrm{~N}_{2} \mathrm{H}_{4}\right)\right] \cdot 2 \mathrm{H}_{2} \mathrm{O}$

XRD-data of complexes: The powder XRD patterns with their d-spacings are given in Table-4. They imply that each set of complexes with similar composition possesses isomorphism among them.
All the complexes were found to be crystalline even though compounds of 2-acambH show more crystallinity.

SEM-EDX studies: The SEM-EDX images of metal oxides of the complexes $\left[\mathrm{Co}(2 \text {-acamb })_{2}\left(\mathrm{~N}_{2} \mathrm{H}_{4}\right)\right] \cdot \mathrm{H}_{2} \mathrm{O},[\mathrm{Cd}(3-$ acamb $\left.)_{2}\left(\mathrm{~N}_{2} \mathrm{H}_{4}\right)\right] \cdot 2 \mathrm{H}_{2} \mathrm{O}$ and $\left[\mathrm{Zn}(4-\mathrm{acamb})_{2}\left(\mathrm{~N}_{2} \mathrm{H}_{4}\right)\right] \cdot 2 \mathrm{H}_{2} \mathrm{O}$ are given as representative examples in Figs. 7-12. These metal oxides are obtained by incinerating the corresponding complexes at their decomposition temperature followed by sintering them for $0.5 \mathrm{~h}$. They have yielded probably nanosized metal oxides.

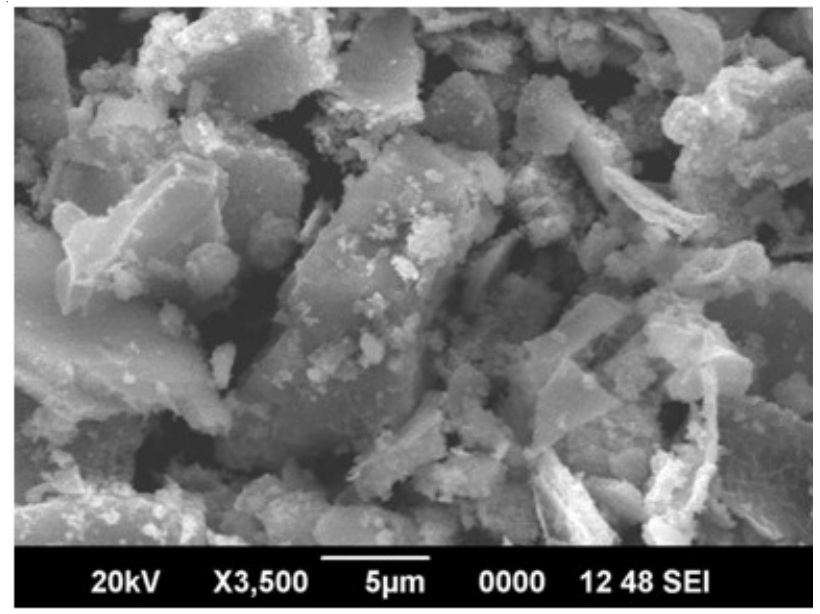

Fig. 7. SEM image of $\mathrm{CoO}$ obtained from $\left[\mathrm{Co}(2 \text {-acamb })_{2}\left(\mathrm{~N}_{2} \mathrm{H}_{4}\right) \cdot \mathrm{H}_{2} \mathrm{O}\right.$

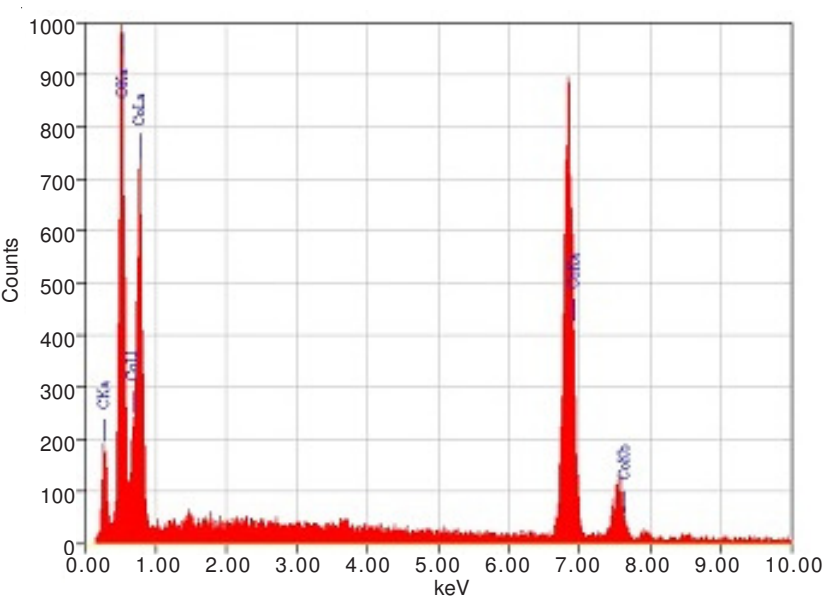

Fig. 8. SEM-EDX picture of $\mathrm{CoO}$

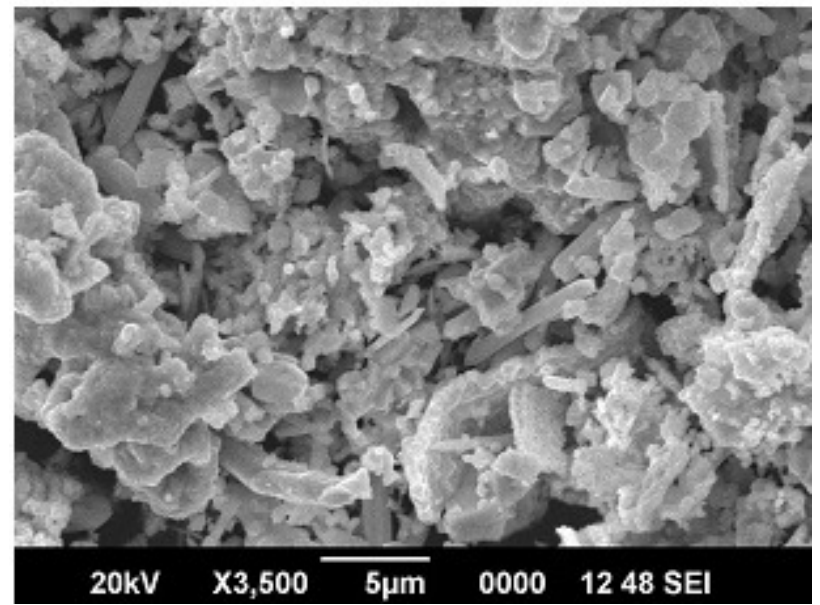

Fig. 9. SEM image of $\mathrm{CdO}$ obtained from $\left[\mathrm{Cd}(3 \text {-acamb })_{2}\left(\mathrm{~N}_{2} \mathrm{H}_{4}\right)\right] \cdot 2 \mathrm{H}_{2} \mathrm{O}$ 


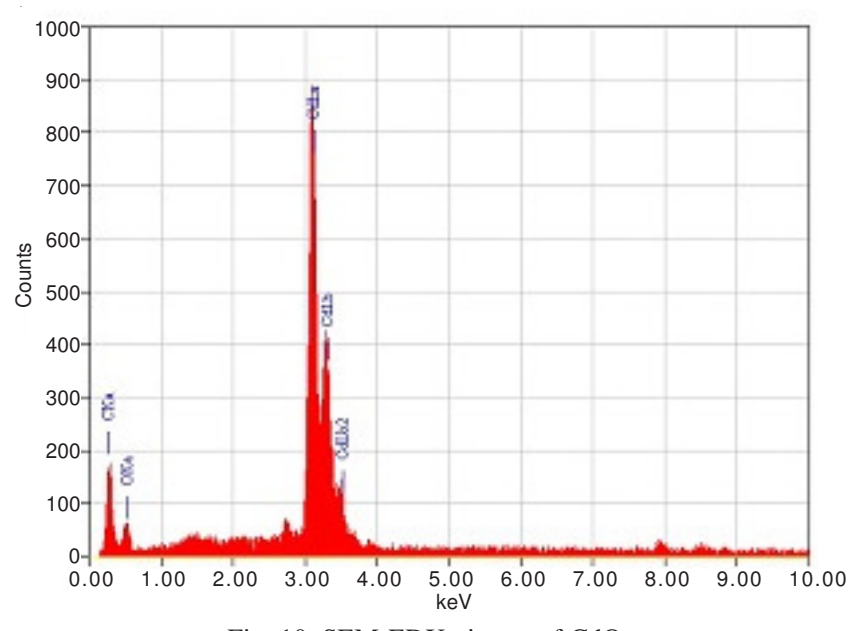

Fig. 10. SEM-EDX picture of CdO

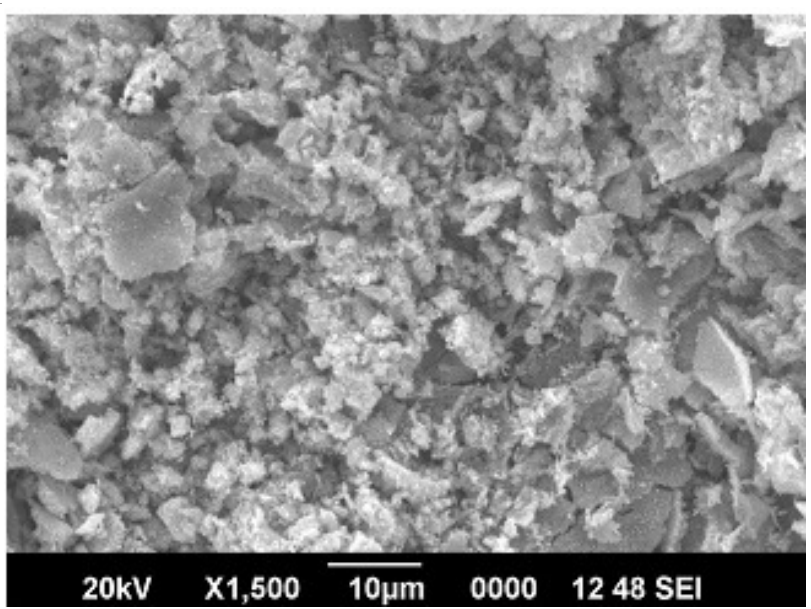

Fig. 11. SEM image of $\mathrm{ZnO}$ obtained from $\left[\mathrm{Zn}(4-\mathrm{acamb})_{2} \cdot \mathrm{N}_{2} \mathrm{H}_{4}\right]_{2} \mathrm{H}_{2} \mathrm{O}$

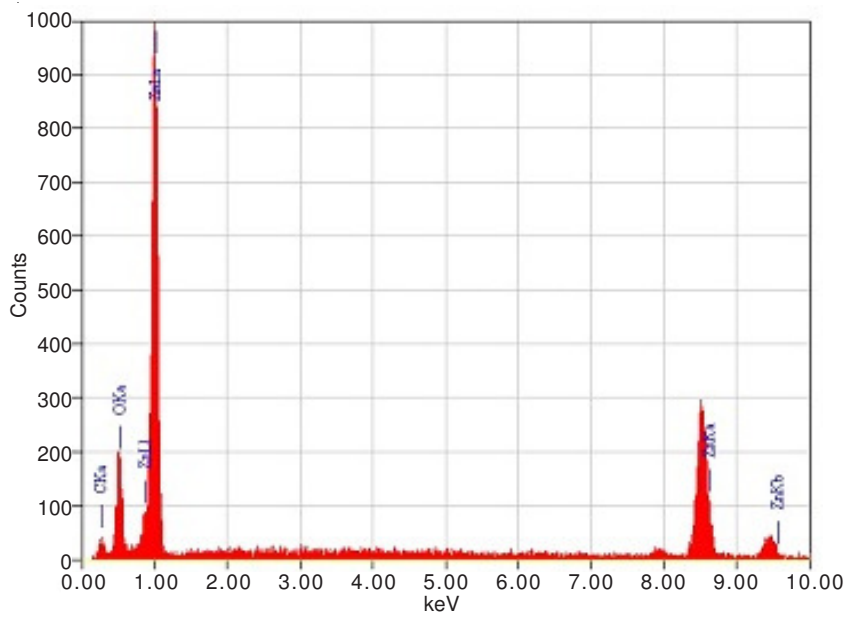

Fig. 12. SEM-EDX picture of $\mathrm{ZnO}$

\section{Conclusion}

All the three isomeric acetamido benzoic acids have formed two categories of hydrated neutral hydrazine complexes with the formulae $\left[\mathrm{M}\{(2-),(3-) \text { and (4-) acamb) }\}_{2}\left(\mathrm{~N}_{2} \mathrm{H}_{4}\right)\right] \cdot \mathrm{xH}_{2} \mathrm{O}$ where 2-acambH $=2$-acetamido benzoic acid, $\mathrm{M}=\mathrm{Ni}$ and $\mathrm{x}=$ 2; $\mathrm{M}=\mathrm{Co}, \mathrm{Cd}$ and $\mathrm{Zn}$ and $\mathrm{x}=1,3$-acambH $=3$-acetamido benzoic acid, $\mathrm{M}=\mathrm{Ni}, \mathrm{Co}$ and $\mathrm{x}=1 ; \mathrm{M}=\mathrm{Cd}$ and $\mathrm{Zn}$ and $\mathrm{x}=2$ and 4-acambH $=4$-acetamido benzoic acid, $\mathrm{M}=\mathrm{Co}$ and $\mathrm{x}=$
$1 ; \mathrm{M}=\mathrm{Ni}, \mathrm{Cd}$ and $\mathrm{Zn}$ and $\mathrm{x}=2$ The $\mathrm{NHCOCH}_{3}$ group is both larger and less strongly electron donating than the $\mathrm{NH}_{2}$ group and hence has not involved in complexation.

The IR frequencies reveal the coordination of the hydrazine with metal and the coordination mode of the carboxylate ion to metal. XRD patterns show isomorphism among the complexes with similar molecular formulae. The SEM-EDS studies confirm the formation of respective metal oxides. The thermal data reveals that various steps of decomposition of the compounds to form their metal oxide. The electronic spectra and the magnetic susceptibility values reveal the coordination and geometry. They suggest that $\mathrm{Ni}, \mathrm{Co}, \mathrm{Cd}$ and $\mathrm{Zn}$ complexes possesses distorted octahedral geometry with coordination number ${ }^{6}$. The proposed structures of these complexes are represented in the Figs. 13-15. However, they may be confirmed by single crystal XRD only.

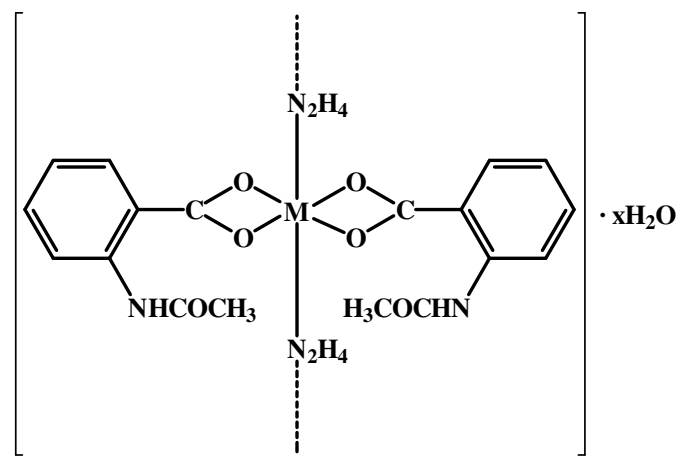

Fig. 13. Proposed structures of transition metal complexes of 2-acetamido benzoate with formula $\left[\mathrm{M}(2 \text {-acamb })_{2}\left(\mathrm{~N}_{2} \mathrm{H}_{4}\right)\right] \cdot \mathrm{xH}_{2} \mathrm{O}$, where $\mathrm{M}=\mathrm{Ni}$ and $\mathrm{x}=2 ; \mathrm{M}=\mathrm{Co}, \mathrm{Cd}, \mathrm{Zn}$ and $\mathrm{x}=1$

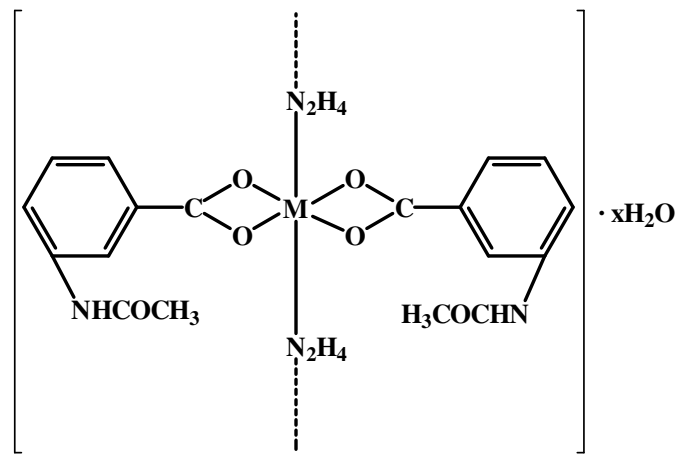

Fig. 14. Proposed structures of transition metal complexes of 3-acetamido benzoate with formula $\left[\mathrm{M}(3 \text {-acamb })_{2}\left(\mathrm{~N}_{2} \mathrm{H}_{4}\right)\right] \cdot \mathrm{xH}_{2} \mathrm{O}$, where $\mathrm{M}=\mathrm{Ni}$, Co and $\mathrm{x}=1 ; \mathrm{M}=\mathrm{Co}, \mathrm{Cd}, \mathrm{Zn}$ and $\mathrm{x}=2$

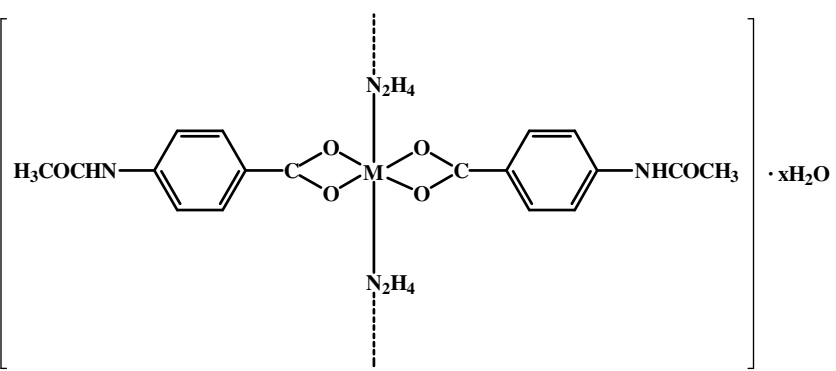

Fig. 15. Proposed structures of transition metal complexes of 4-acetamido benzoate with formula $\left[\mathrm{M}(4 \text {-acamb })_{2}\left(\mathrm{~N}_{2} \mathrm{H}_{4}\right)\right] \cdot \mathrm{xH}_{2} \mathrm{O}$, where $\mathrm{M}=\mathrm{Ni}$, $\mathrm{Cd}, \mathrm{Zn}$ and $\mathrm{x}=2 ; \mathrm{M}=\mathrm{Co}$ and $\mathrm{x}=1$ 
XRD DATA OF COMPLEXES (d-SPACING IN Å UNITS AND INTENSITY IN PARENTHESES)

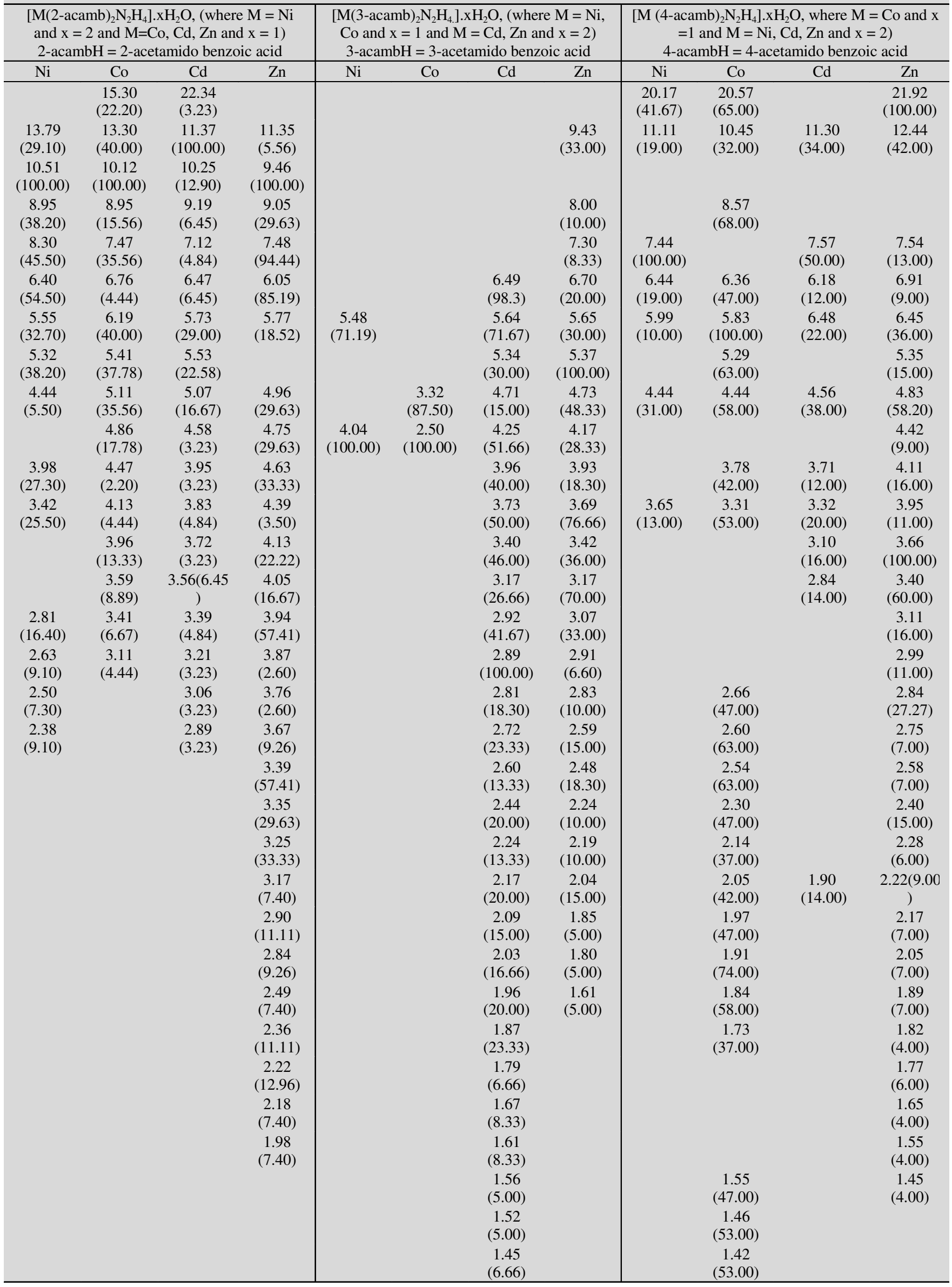




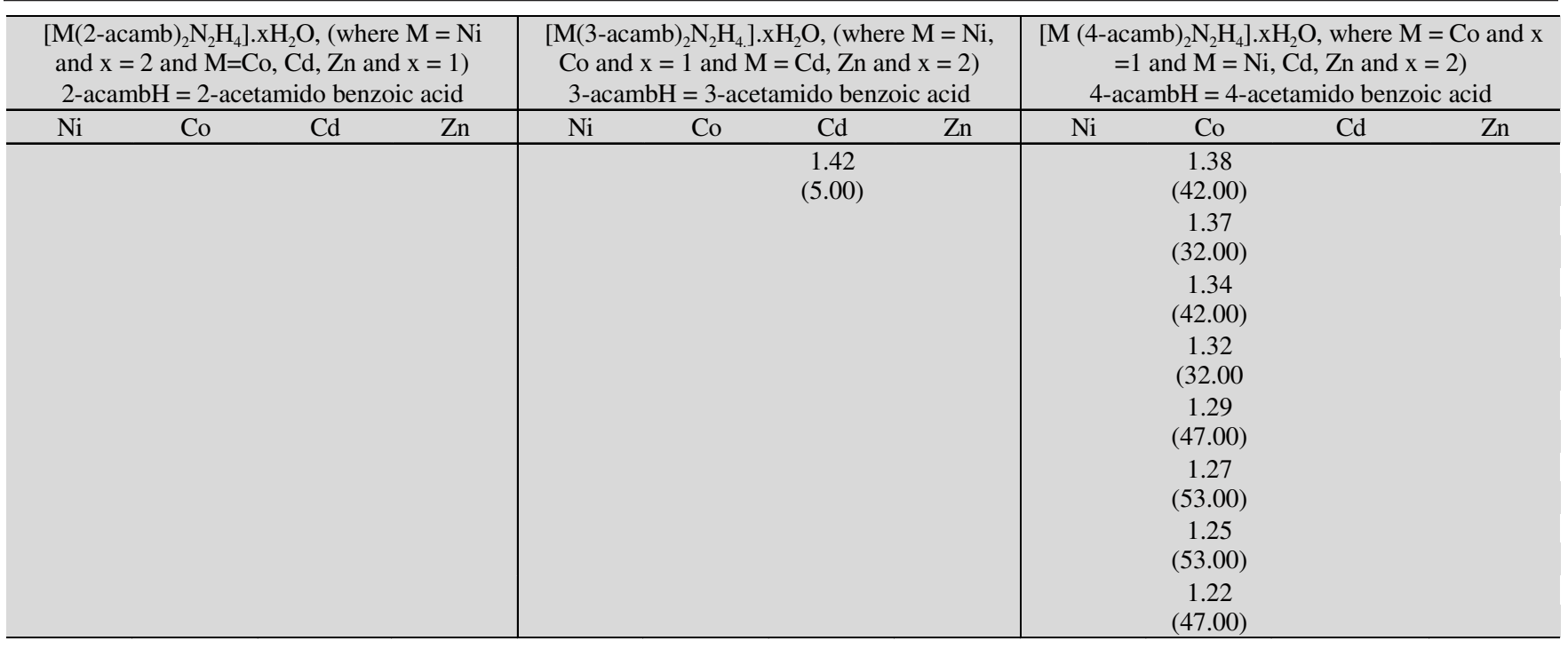

\section{ACKNOWLEDGEMENTS}

The authors acknowledged to All India Council for Technical education (AICTE) for granting the financial support for this work (grant in aid No./8023/BOR/RID/RPS- 2, 2008-2009).

\section{REFERENCES}

1. B.N. Sivasankar and S. Govindarajan, J. Therm. Anal. Calorim., 48, 1401 (1997).

2. S. Yasodhai and S. Govindarajan, Synth. React. Inorg. Met.-Org. Chem., 30, 745 (2000).

3. K. Kuppusamy and S. Govindarajan, Synth. React. Inorg. Met.-Org. Chem., 26, 225 (1996).

4. K. Kuppusamy and S. Govindarajan, Eur. J. Solid State Inorg. Chem., 32, 997 (1995).

5. S. Vairam, T. Premkumar and S. Govindarajan, J. Therm. Anal. Calorim., 100, 955 (2010).

6. S. Vairam, T. Premkumar and S. Govindarajan, J. Therm. Anal. Calorim., 101, 979 (2010).

7. D. Gajapathy, S. Govindarajan, K.C. Patil and H. Manohar, Polyhedron, 2, 865 (1983).

8. S. Govindarajan, K.C. Patil, M.D. Poojary and H. Manohar, Inorg. Chim. Acta, 120, 103 (1986).

9. T. Premkumar and S. Govindarajan, J. Therm. Anal. Calorim., 74, 325 (2009).
10. T. Premkumar and S. Govindarajan, J. Therm. Anal. Calorim., 79, 115 (2005).

11. N. Arunadevi and S. Vairam, E.-J. Chem., 6(S1), S413 (2009).

12. T. Premkumar and S. Govindarajan, Thermochim Acta, 386, 35 (2002).

13. T. Premkumar and S. Govindarajan, J. Therm. Anal. Calorim., 84, 395 (2006).

14. L. Vikram and B.N. Sivasankar, J. Therm. Anal. Calorim.,91, 963 (2008).

15. B. Raju and B.N. Sivasankar, J. Therm. Anal. Calorim., 94, 289 (2008).

16. X. Yin, J. Fan, Z. Wang and W.G. Zhang, Z. Anorg. Allg. Chem., 637, 773 (2011)

17. Z.H. Wang, J. Fan and W.G. Zhang, Z. Anorg. Allg. Chem., 635, 2333 (2009).

18. W. Li, C.H. Li, Y.Q. Yang and Y.F. Kang, Chin. J. Inorg. Chem., 23, 2023 (2007).

19. Y.B. Wang, X.J. Zheng, W.J. Zhuang and L.P. Jin, Eur. J. Inorg. Chem., 2003, 3572 (2003).

20. I.A. Vogel, A Text Book of Quantitative Inorganic Analysis, Longmans., London, p. 380 and 433 (1975).

21. A.B.P. Lever, Inorganic Electronic Spectroscopy, Elsevier, Amsterdam, (1984).

22. K. Nakamoto, A Text book of Infrared and Raman Spectra of Inorganic and Coordination Compounds, John Wiley and Sons, USA, pp. 228229 (1986).

23. R.C. Weast, CRC Hand Book of Chemistry and Physics, CRC Press: Cleveland, Ohio (1976/1977).

24. D.V. Junior, J.W. Tilley and R.A. Lemahieu, J. Org. Chem., 46, 4614 (1981). 